\title{
Screening for Risk of Rehospitalization from Home Care: Use of the Outcomes Assessment Information Set and the Probability of Readmission Instrument
}

\author{
Kathryn H. Bowles, ${ }^{1 *}$ Jacqueline R. Cater $^{2 \dagger}$ \\ ${ }^{1}$ School of Nursing, University of Pennsylvania, 420 Guardian Drive, Philadelphia, PA 19104-6096 \\ ${ }^{2}$ Biomedical Statistical Consulting, 1357 Garden Road, Wynnewood, PA 19096 \\ Received 22 August 2002; accepted 10 December 2002
}

\begin{abstract}
The purpose of this study was to evaluate the Outcomes Assessment Information Set (OASIS) compared with the Probability of Readmission $\left(P_{r a}\right)$ instrument for use in predicting rehospitalization during home care. Using logistic regression and receiver operating characteristic (ROC) curve analysis, the $\mathrm{P}_{\text {ra }}$ instrument was found to be significantly better at predicting rehospitalization than the OASIS case mix weight, clinical, or service scores. The area under the curve (AUC) for the $P_{\text {ra }}$ was .686 compared with .549 for the OASIS case mix weight $(p=.010)$. Similar results were found for the OASIS clinical and service scores. The AUC for the function score of $\geq 2$ (.599) provided the closest approximation to the $P_{\text {ra }}(.686)$, and the difference between the two was not statistically significant $(p=.120)$. The OASIS function score could be used to identify at-risk home care patients without having to also use the $P_{\text {ra }}$ instrument. (c) 2003 Wiley Periodicals, Inc. Res Nurs Health 26:118-127, 2003
\end{abstract}

Keywords: home health care; hospital readmission; OASIS; $\mathrm{P}_{\mathrm{ra}}$

The current financial and manpower crises in health care require that clinicians and administrators carefully assign their scarce patient care resources to achieve the best outcomes. One desirable skill for those concerned with quality and cost is the ability to predict and prevent rehospitalization. Referral for skilled home care nursing on hospital discharge is one way to meet patients' continuing care needs and prevent rehospitalization (Martens \& Mellor, 1997; Proctor, MorrowHowell, Li, \& Dore, 2000).

Once a referral to home care is made, many home care agencies at present lack a systematic, reliable method to identify patients at risk of rehospitalization. Generally, plans of care are based on individual clinical judgment and prescribed

Contract grant sponsor: Edna G. Kynett Memorial Foundation, Philadelphia, PA.

Correspondence to Kathryn H. Bowles, School of Nursing, University of Pennsylvania,

420 Guardian Drive, Philadelphia, PA 19104-6096.

*Assistant Professor.

†Biostatistician.

Published online in Wiley InterScience (www.interscience.wiley.com)

DOI: 10.1002/nur.10071 
clinical pathways that do not differentiate between those who may need more intense services and those who may need less intense services to produce optimal clinical and financial outcomes. The ability to identify patients likely to benefit from specialized interventions would enhance care planning to prevent costly rehospitalization. For example, specific, targeted interventions such as care protocols provided by advanced practice nurse specialists could successfully prevent rehospitalization of at risk patients (Naylor et al., 1994, 1999).

The aim of this study was to compare the efficacy of the routinely collected Outcomes Assessment Information Set (OASIS) with the Probability of Readmission $\left(\mathrm{P}_{\mathrm{ra}}\right)$ scale in screening patients for risk of hospital readmission during home care. All Medicare-certified home care agencies are mandated by the Centers for Medicare and Medicaid Services (CMS) to collect OASIS data on all Medicare patients. The OASIS is widely used in home care for assessment, care planning, monitoring, case mix adjustment, and outcomes measurement (Shaughnessy, Crisler, \& Schlenker, 1998), and CMS requires it to determine the rate of reimbursement for a particular patient and quality outcomes for agencies. However, it was not designed to predict rehospitalization rates. In outpatient settings the Probability of Readmission $\left(\mathrm{P}_{\mathrm{ra}}\right)$ instrument is widely used for this purpose (Boult et al., 1993). The OASIS and $\mathrm{P}_{\mathrm{ra}}$ contain many patient characteristics associated with readmission risk, such as severity of illness, medical diagnosis, functional status, social support, age, and prior resource use. It would be of added value if the OASIS data, already routinely collected, could also be used to guide clinical decision making by identifying patients at risk for rehospitalization, without adding another instrument such as the $\mathrm{P}_{\text {ra. }}$.

In addition to promoting better outcomes for the patient, preventing hospital readmission helps to avoid negative consequences for the home care agency. Under the home care prospective payment system the agency receives a sum of money to care for a patient for 60 days based on the results of the OASIS completed on admission. If a patient is readmitted to the hospital from home care, the home care nurse must repeat the lengthy OASIS on discharge from home care to the hospital and again at readmission to home care. The agency also risks losing the patient to another agency on discharge, with a possible loss of the full 60-day Medicare reimbursement. Further, a hospital admission is costly, requiring additional Medicare reimbursement, with the concomitant implications for qua- lity (Fortinsky, Madigan, \& Tullai-McGuinness, 2000). It would be beneficial for the patient and the home care agency to be able to identify patients at risk for rehospitalization in order to target expertise and other resources to meet care needs effectively.

The identification of patients at risk for poor discharge outcomes such as rehospitalization is an important area of research and of evidence-based practice. Many researchers have identified the characteristics of at-risk patients including age $>70$, female gender, chronic cardiac or respiratory problems, widowed, cognitive impairment, polypharmacy, poor self-rated health, and long length of stay or complications during a hospital stay (Anderson, Helms, Hanson, \& DeVilder, 1999; Berkman, Walker, Bonander, \& Holmes, 1992; Bowles, Naylor, \& Foust, 2002; Kellogg, Brickner, Conley, \& Conroy, 1991; Lagoe, Noetscher, \& Murphy, 2001; Leiby \& Shupe, 1992; Naylor et al., 1994). The level of dependence in activities of daily living (ADLs) such as mobility, eating, bathing, and feeding is also a predictor of risk of rehospitalization (Redeker \& Brassard, 1996; Reed, Buckner, \& Pearlman, 1991). Prior hospitalization has consistently been a strong predictor of subsequent hospitalization (Corrigan \& Martin, 1992; Kellogg et al., 1991; Reed et al., 1991; Soeken, Prescott, Herron, \& Creasia, 1991; Vinson, Rich, Sperry, Shah, \& McNamara, 1990).

Research on readmission for patients receiving skilled home care has been limited but is growing. Hospital readmission during home care was found to be more likely for cardiac surgery patients who had complications during their inpatient stay or who had functional deficits in ADLs (Redeker \& Brassard, 1996). Number of medications (seven or more) was similarly associated with increased risk of rehospitalization for patients older than 65 (Flaherty, Perry, Lynchard, \& Morley, 2000), as were reported previous hospitalizations, use of home health aide services, and short length of home care stay (Hoskins, Walton-Moss, Clark, Schroeder, \& Thiel, 1999). Anderson and colleagues (1999) found that chronic illness, including congestive heart failure (CHF), was the best indicator of hospital readmission from home care, and Happ, Naylor, and Roe-Prior (1997) found that social and behavioral factors contributed to the rehospitalization of heart failure patients. Other factors such as advanced patient age, stage or severity of illness, functional dependence, prior hospitalization, emergent admission, type of and length of care after discharge, numerous medications, and mobility status were also associated 
with readmission of CHF patients (Burns \& Nichols, 1991; Hoskins et al., 1999; Shipton, 1996, 1997). In addition, home care patients consistently experienced readmission rates $33 \%$ higher than other patients discharged from hospitals, making it even more important to identify those at risk (Lagoe et al., 2001).

Although much is known about individual characteristics associated with hospitalization, this knowledge has not been synthesized into a standardized instrument that could be used in home care to identify patients at risk for rehospitalization. Results of the present study could provide guidance about using the OASIS and $\mathrm{P}_{\mathrm{ra}}$ instruments to identify patients at risk for rehospitalization and subsequently to match them with a targeted plan of home care.

\section{METHOD}

\section{Design}

A cross-sectional design was used to compare the OASIS and $\mathrm{P}_{\mathrm{ra}}$ instruments in screening patients for risk of rehospitalization while receiving a 60-day episode of skilled home care.

\section{Sample}

The sample of 147 patients was obtained from a primary study comparing the effects on rehospitalization of traditional skilled home care $(n=88)$ with two types of telehome care: home care with televideo visits $(n=24)$ or home care with telephone follow-up $(n=35)$. The university institutional review board and the home care agency approved the primary study. All identifying information was removed, and no new information was collected for this analysis; therefore, this secondary analysis met the criteria for exemption from further review, as determined by the institutional review board.

The criteria for eligibility to participate in the study were: age 65 or older; admitted to a large urban home care agency in the Northeast with a primary or coexisting diagnosis of CHF; able to hear, see, and answer questions; having a telephone in the home; and being insured by Medicare. All eligible patients were assessed using the OASIS, as is the usual practice in home care. They were also screened using the $\mathrm{P}_{\mathrm{ra}}$ instrument in order to stratify them into risk groups for the primary study. This provided a sample of patients with data from both screening instruments. The entire sample was $70 \%$ female, $42 \%$ Black, $21 \%$ Caucasian, and $1 \%$ Hispanic; the average age was 78 . The majority of patients, $77 \%$, rated their health as fair or poor, and $29 \%$ were rehospitalized within 60 days of home care admission. The average $\mathrm{P}_{\mathrm{ra}}$ score was .52 (range of .15-.75); and the average case mix weight was 1.39 (range .522.86). The majority had clinical OASIS scores showing minimum or low severity; $35.5 \%$ had a score of 0 (minimum) and $40.6 \%$ a score of 1 (low). More than two thirds (67\%) scored $\geq 2$ (moderate severity or worse) for function. The service domain showed that $90 \%$ of the sample scored 2 (moderate) and below.

\section{Instruments}

Oasis. Since October 2000 the Health Care Financing Administration (HCFA), now known as the Centers for Medicare and Medicaid Services (CMS), has mandated collection of the OASIS from all Medicare patients on home care admission, transfer, and discharge and every 62 days if the patient remains in home care. The OASIS is an instrument used by home care clinicians to assess patient status in three domains, using 14 clinical items (medical diagnoses, services received in home, vision, pain, wounds, multiple pressure ulcers, stage and status of ulcers and/or wounds, shortness of breath, incontinence, presence of an ostomy, and behavioral problems), six functional items (dressing upper and lower body, bathing, toileting, transferring, and locomotion), and two service-related items (origin of discharge prior to home care and need for physical therapy; Shaughnessy et al., 1998). The OASIS assessment categorizes patients according to severity levels in each domain. The clinical and service domains each have four levels: minimum (0), low (1), moderate (2), and high (3). The functional domain has five levels: minimum (0), low (1), moderate (2), high (3), and max (4). The domain scores also generate a case mix weight set by CMS that is used to calculate the amount of reimbursement received by the agency. It ranges from .5265 to 2.8113 , with higher scores associated with poorer clinical, functional, and service scores. Each of the domain scores and the case mix weight were used in this analysis as predictor variables for rehospitalization.

The staff educator at the home care agency used case studies to train registered nurses and physical therapists in how to use the OASIS instrument. Individual managers observed their assessments in the patients' homes to determine adequate 
consistency in scoring. The agency nurses who participated in this study achieved interrater reliability ratings of $83 \%$. Neal (2000) reported an interrater reliability of .60, and Madigan \& Fortinsky (2000) achieved internal consistency of .86 and .91, respectively, on admission and discharge for the functional domain. Nunnally and Bernstein (1994) suggested that an alpha of at least .70 is acceptable for a new scale. Shaughnessy, Schenkler, and Hittle (1995) reported kappa scores on the functional domain items ranging from .61, for feeding, to .78, for grooming. Madigan \& Fortinsky also reported kappa scores of .41 to 1.0 for functional domain items and .54 to 1.0 for clinical domain items. Kappa scores provide a measure of the extent of agreement beyond chance (Brennan \& Hays, 1992). Moderate (.41-.60), substantial $(.61-.80)$, or almost perfect scores $(>.81)$ are considered acceptable (Kerr, 1994).

$\boldsymbol{P}_{\boldsymbol{r a}}$ instrument. The $\mathrm{P}_{\mathrm{ra}}$ instrument is an eightitem questionnaire used in research and clinical practice to assess factors shown to predict rehospitalization more than once within four years (Boult et al., 1993). The patient's answers to eight questions on demographics (age, gender), health (self-rated health, presence of diabetes and heart disease), social support (presence of a caregiver), and service utilization (number of doctor visits and hospitalizations in last 12 months) were scored according to a logistic equation. The resulting $\mathrm{P}_{\text {ra }}$ score was a value between 0 and 1 , with higher values indicating higher probability of readmission. In other studies it was found that Medicaid patients (Pacala, Boult, \& Boult, 1995) and managed care patients (Pacala, Boult, Reed, \& Aliberti, 1997), with $P_{\text {ra }}$ scores $\geq .50$ were rehospitalized at twice the rate of patients scoring $<.50$. The $\mathrm{P}_{\text {ra }}$ instrument has been used to identify communitydwelling patients at risk of rehospitalization or to target special services (Pacala et al., 1995; Boult, Pacala, \& Boult, 1995). A high test-retest reliability $(k=.78)$ has been reported by others (Boult, Boult, Pirie, \& Pacala, 1994) and was not repeated for this study. However, interrater reliabilities were checked during training between the principal investigator (PI) and the project manager who administered the instrument for five patients with $100 \%$ agreement, and the procedure for calculating the $\mathrm{P}_{\text {ra }}$ score was spot-checked each month by the PI during the study period.

\section{Procedure}

Based on the usual home care admission procedures, all patients were assessed in person by the admitting registered nurses using the OASIS instrument. Each day the registered nurse project manager of the primary study checked the admission $\log$ for patients 65 and older admitted with $\mathrm{CHF}$ as one of the top three admitting diagnoses. Eligible patients were called or visited, the study was explained to them, and their verbal permission to be screened using the $\mathrm{P}_{\mathrm{ra}}$ was obtained. Answers to the eight questions were obtained and scored by the nurse project manager according to a logistic regression equation to calculate the $\mathrm{P}_{\mathrm{ra}}$ score (Boult et al., 1993). Patients were asked to consent for the primary study. Those who scored .50 or higher on the $\mathrm{P}_{\mathrm{ra}}$ were randomized to receive a form of telehome care, either home care plus video visits or home care plus telephone reassurance calls. Patients with scores less than .50 or those who were not eligible for the telehome care groups (e.g., hard of hearing, blind, no phone, cognitively impaired) received the usual home care. For this study patient data from all groups were combined, and any differences between groups were statistically controlled as described below. The project manager collected data on rehospitalization from a patient's home care record at the end of the 60-day episode. This procedure provided data from both instruments on all study participants.

\section{Data Analysis}

For the first part of the analysis, the demographic characteristics of the telehome care and usual care groups were compared. In all statistical models used in the present study, a term for group (telehome care vs. usual care) was included in each model in order to control for the effect of group membership on the predictive validity of each instrument for rehospitalization.

Second, the performance of the $\mathrm{P}_{\mathrm{ra}}$ score and the OASIS case mix weight, clinical, functional, and service scores was assessed for accuracy in predicting rehospitalization. Statistical significance was set at $p<.05$ (in figures significance $<.01$ is indicated by two asterisks $\left.\left[{ }^{* *}\right]\right)$. A series of logistic regressions were performed to measure the area under the curve (AUC) for the receiver operating characteristic (ROC) curve for each diagnostic test. This was done separately for each of the domain scores of the OASIS (clinical, function, and service, as well as case mix weight) and for the overall $\mathrm{P}_{\mathrm{ra}}$ score. A ROC curve plots the sensitivity of the test (the proportion of true positives, or accurately predicting a rehospitalization) versus specificity (the proportion of false positives, or 
predicting a rehospitalization when the patient was actually not rehospitalized) over a series of cut points. When the predictive accuracy of the test is equal to chance (a $50 \%$ success score), the ROC curve lies along a 45-degree line (the dotted line on the graph, Fig. 1). As the accuracy of the test improves, the ROC curve moves up and to the left from the 45-degree line. The area under the ROC curve (AUC) measures the probability that a randomly selected participant who actually was rehospitalized has a greater probability of being classified as a rehospitalization compared with a randomly selected participant who was not rehospitalized. The AUC is therefore a summary measure of predictive value across all possible cut points of the prediction index.

Third, the ROC curve for the $\mathrm{P}_{\mathrm{ra}}$ instrument was compared to the ROC curve for each domain of the OASIS instrument to assess relative differences in the ability to predict rehospitalizations using a test for correlated data (DeLong, DeLong, \& ClarkPearson, 1988). Finally, sensitivity and specificity estimates were computed for cut points of the $\mathrm{P}_{\mathrm{ra}}$ and OASIS scales (McNeil, Keeler, \& Adelstein, 1975; Soz, Blatt, Higgins, \& Marion, 1988).

\section{RESULTS}

Table 1 summarizes the characteristics of the entire sample. A comparison of the telehome care and usual care groups showed that they differed statistically on prior hospitalizations $(p<.001)$, with the telehome care group having more prior hospitalizations (37\% versus $12 \%$, respectively, hospitalized three or more times) and more social support ( $97 \%$ versus $67 \%$, respectively, lived with a caregiver, $p<.0001$ ) as well as more subsequent hospital admissions since home care $(37 \%$ versus $23 \%, p=.05)$. Thus, to control for these differences, a stratified analysis (Fleiss, 1986; Hosmer \& Lemeshow, 1989) was performed by including a term for group in all subsequent models. Because the telehome care group had $\mathrm{P}_{\mathrm{ra}}$ scores restricted to .50 or above, neither a test for interaction between group membership and $\mathrm{P}_{\mathrm{ra}}$ score nor comparative analyses by subgroup (usual or tele-home care) was warranted. Rather, a term for group membership was included in all models.

Figure 1 shows the ROC curves generated by logistic regression for the $\mathrm{P}_{\mathrm{ra}}$ score versus the OASIS case mix weight. The $\mathrm{P}_{\mathrm{ra}}$ instrument was

Table 1. Demographic and Health Characteristics

\begin{tabular}{|c|c|c|}
\hline Variable & Level & $N=147(\%)$ \\
\hline Age & Mean \pm SD & $78.3 \pm 8.85$ \\
\hline \multirow[t]{4}{*}{ Race } & Caucasian & $31(21)$ \\
\hline & African American & $61(42)$ \\
\hline & Hispanic & $1(1)$ \\
\hline & Missing & $54(37)$ \\
\hline \multirow[t]{2}{*}{ Gender } & Female & $103(70)$ \\
\hline & Male & $44(30)$ \\
\hline \multirow[t]{6}{*}{ Perception of health } & Excellent & $1(1)$ \\
\hline & Very good & $4(3)$ \\
\hline & Good & $18(12)$ \\
\hline & Fair & $64(44)$ \\
\hline & Poor & $49(33)$ \\
\hline & Missing & $11(7)$ \\
\hline \multirow[t]{5}{*}{ Hospital in last 12 months } & None & $10(7)$ \\
\hline & 1 time & $43(29)$ \\
\hline & 2-3 times & $50(34)$ \\
\hline & $>3$ times & $31(21)$ \\
\hline & Missing & $13(9)$ \\
\hline \multirow{3}{*}{$\begin{array}{l}\text { Social support } \\
\text { (presence of a caregiver) }\end{array}$} & No & $25(17)$ \\
\hline & Yes & $103(70)$ \\
\hline & Missing & $19(13)$ \\
\hline \multirow[t]{2}{*}{ Hospital admission since home care } & No readmit & $105(71)$ \\
\hline & Readmit & $42(29)$ \\
\hline \multirow[t]{2}{*}{$\mathrm{P}_{\text {ra }}$ score } & $<0.50$ & $48(33)$ \\
\hline & $>0.50$ & $99(67)$ \\
\hline
\end{tabular}


better at predicting rehospitalization than the OASIS case mix weight. The AUC for the $\mathrm{P}_{\mathrm{ra}}$ instrument was .686, compared with an AUC of .549 for the OASIS case mix weight. A power analysis showed a power of .75 to detect an AUC of at least .58, given the empirical $\mathrm{P}_{\mathrm{ra}}$ estimate of .69 (Obuchowski, 1994). The ROC curve for the OASIS case mix weight indicated that its ability to predict rehospitalization was not much better than chance. In addition, a test for differences in ROC curves for correlated data showed that the difference in AUCs was statistically significant $(p=.01)$ between the two instruments. Similar results were found for the OASIS clinical and service scores, as can be seen in Figures 2 and 3.

The OASIS function score most closely approximated the ability of the $\mathrm{P}_{\mathrm{ra}}$ instrument to predict rehospitalizations (Fig. 4). The AUC for the function score (.599) was closest to the AUC for the $\mathrm{P}_{\mathrm{ra}}$ score (.686), and the difference between the two ROC curves was not statistically significant $(p=.12)$. This relative similarity in predicting rehospitalizations was also supported by the sensitivity and specificity for various cut points for each of the instruments, which are summarized in Table 2. It appeared that using a cut point $\geq 2$ for the OASIS function score resulted in sensitivity and specificity estimates similar to those found using the $\mathrm{P}_{\mathrm{ra}}$ instrument. For the $\mathrm{P}_{\mathrm{ra}}$ instrument, a cut point of .50 resulted in sensitivity of 85.7 and specificity of 40.0 . The $\mathrm{P}_{\mathrm{ra}}$ instrument accurately detected $85.7 \%$ of the patients who would be rehospitalized, whereas it identified $1-.40$, or $60.0 \%$, as false positives (identifying patients as at risk for rehospitalization who were not sub- sequently rehospitalized). For the OASIS function score, sensitivity was 77.1, and specificity was 35.9. The OASIS function score accurately detected $77.1 \%$ of the patients who subsequently would be rehospitalized, while identifying $1-.359$, or $64.1 \%$, as false positives.

\section{DISCUSSION}

Although far from perfect, study results suggest that the $\mathrm{P}_{\mathrm{ra}}$ and the function score of the OASIS were helpful in predicting which patients were likely to be rehospitalized. The $\mathrm{P}_{\mathrm{ra}}$ performed better than the OASIS because it was both more sensitive $(85.7 \%)$ and more specific $(40.0 \%)$ in predicting who would be rehospitalized. If agency personnel could obtain the data for the $\mathrm{P}_{\mathrm{ra}}$ on all admissions, the $\mathrm{P}_{\mathrm{ra}}$ might more effectively assist them in identifying patients at risk for rehospitalization. The $\mathrm{P}_{\mathrm{ra}}$ takes approximately $5 \mathrm{~min}$ to complete and another $5 \mathrm{~min}$ to score once the logistic formula is set up in a spreadsheet. However, agencies may feel confident using a functional domain score $\geq 2$ because, although it was not as sensitive $(77.1 \%)$ or specific (35.9\%) as the $\mathrm{P}_{\mathrm{ra}}$, the difference was not statistically significant. Findings suggest clinicians could use the OASIS function score and avoid adding another data collection instrument until a better instrument is developed or this research is further refined.

In general, the $\mathrm{P}_{\mathrm{ra}}$ instrument may have performed better than the OASIS because it included prior hospitalization as one of the more heavily weighted factors in the regression formula that

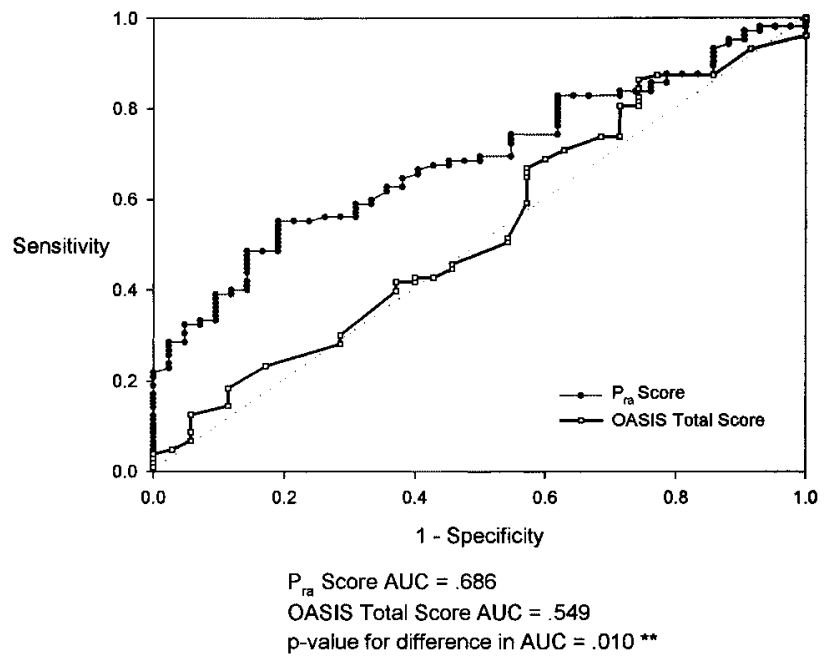

FIGURE 1. ROC curves for $P_{\text {ra }}$ scores and OASIS case mix score $(N=147)$. 


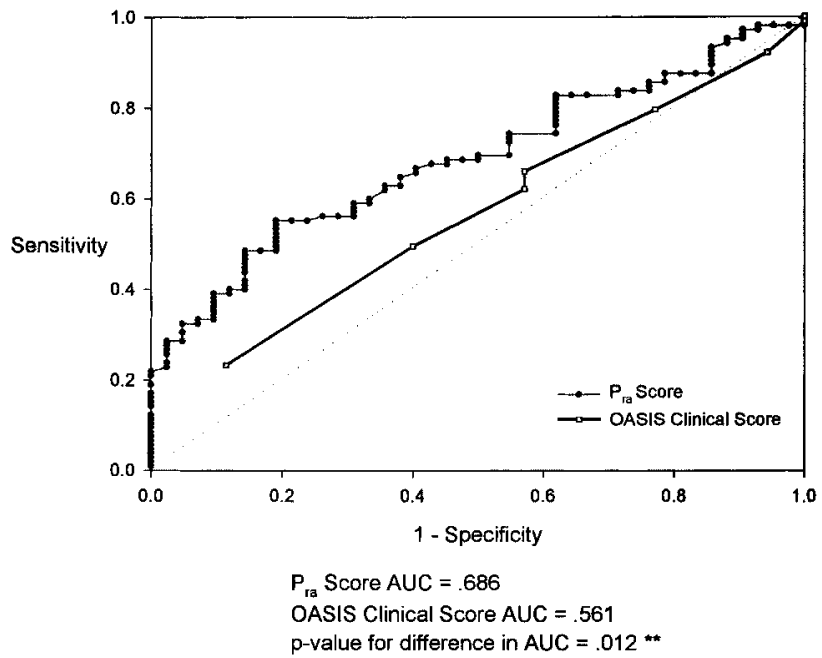

FIGURE 2. ROC curves for $P_{\text {ra }}$ scores and OASIS clinical score $(N=147)$.

determined the $\mathrm{P}_{\mathrm{ra}}$ score. Several investigators have shown the importance of prior hospitalization in predicting the likelihood of future hospitalization (Corrigan \& Martin, 1992; Kellogg et al., 1991; Reed et al., 1991; Soeken et al., 1991; Vinson et al., 1990). In the OASIS prior hospitalization results in a lower score than if a patient came from a skilled nursing facility or a rehabilitation facility. Therefore, the effect of prior hospitalizations is attenuated in the OASIS score.

The OASIS domains and case mix weight used in this study do not include all the items in the full OASIS instrument, which includes other clinical, financial, family, social, and environmental factors comparable to those that Winograd et al. (1991) and Soeken and colleagues (1991) suggested may play a role in predicting hospital utilization. Perhaps the predictive value of the OASIS would improve if the additional items were analyzed versus the case mix weight and clinical, function, and service domain scores. Only the domain and case mix scores were used in this study because we believe that, unlike the individual OASIS items, these summary scores would be more convenient and readily available for a clinician to use in practice. One potential study that could build on this one might be an analysis that included all the OASIS items for patients with function scores of 2 or more. Such an analysis might identify a more defined set of factors, which could result in

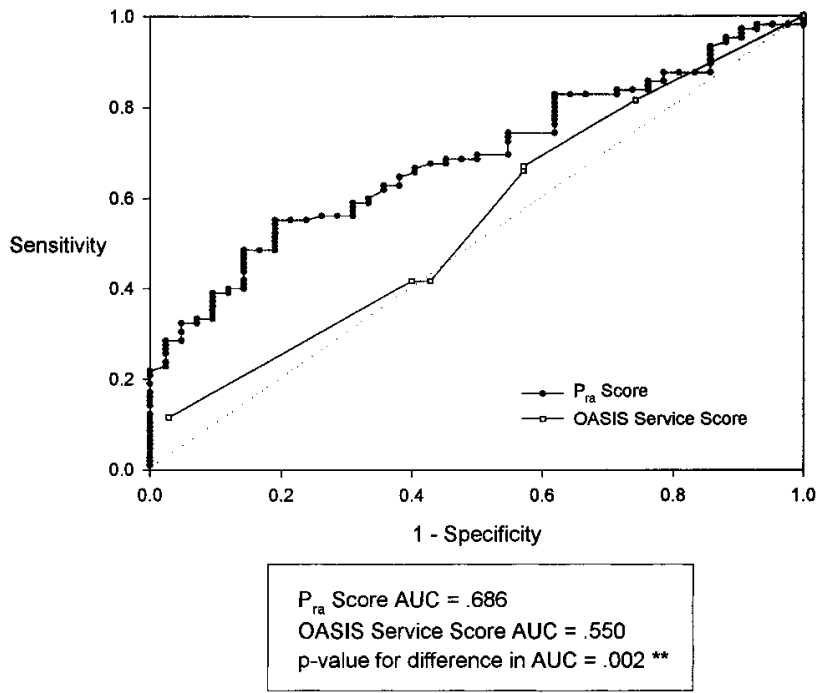

FIGURE 3. ROC curves for $P_{\text {ra }}$ scores and OASIS service score $(N=147)$. 


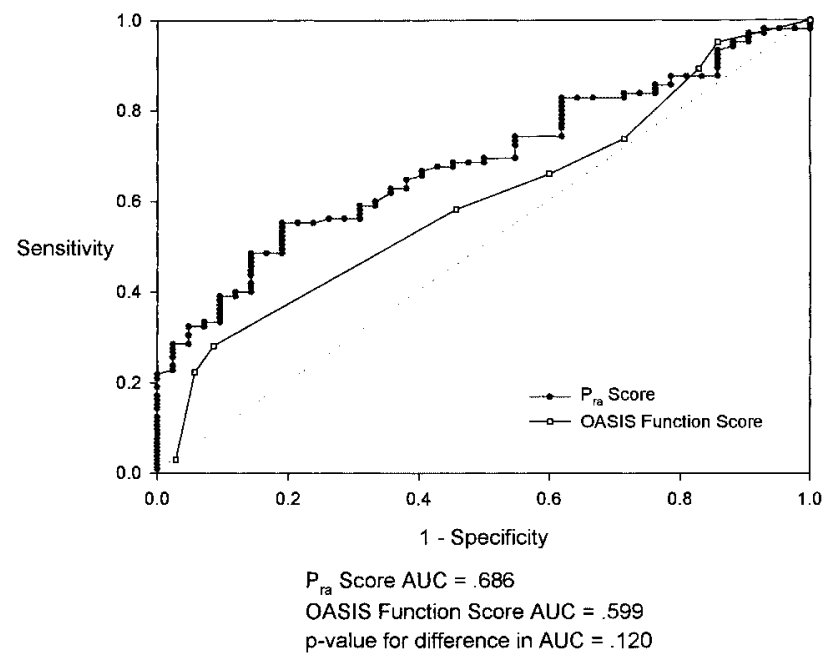

FIGURE 4. ROC curves for $P_{\text {ra }}$ scores and OASIS function score $(N=147)$.

Table 2. Comparison of Sensitivity and Specificity for Cut Points of $P_{r a}$ and OASIS Instruments $(N=147)$

\begin{tabular}{lccc}
\hline & $\begin{array}{c}\text { Cut } \\
\text { Point }\end{array}$ & Sensitivity & Specificity \\
\hline & 30 & 100.0 & 7.6 \\
& 35 & 100.0 & 10.5 \\
& 40 & 100.0 & 17.1 \\
& 45 & 95.2 & 28.6 \\
& 50 & 85.7 & 40.0 \\
& 55 & 59.9 & 64.8 \\
& 60 & 38.1 & 77.1 \\
& 65 & 19.1 & 89.5 \\
OASIS clinical & 70 & 4.8 & 97.1 \\
& 75 & 2.4 & 99.1 \\
& $\geq 1$ & 68.6 & 36.9 \\
OASIS function & $\geq 2$ & 22.9 & 75.7 \\
& $\geq 3$ & 0.0 & 95.2 \\
& $\geq 1$ & 94.3 & 8.7 \\
& $\geq 2$ & 77.1 & 35.9 \\
OASIS service & $\geq 3$ & 28.6 & 81.6 \\
& $\geq 4$ & 2.9 & 94.2 \\
& $\geq 1$ & 60.0 & 42.7 \\
OASIS case mix & $\geq 2$ & 57.1 & 42.7 \\
& $\geq 3$ & 2.9 & 87.4 \\
& $\geq 0.8$ & 74.3 & 29.1 \\
& $\geq 0.9$ & 62.9 & 35.9 \\
& $\geq 1.0$ & 60.0 & 40.8 \\
& $\geq 1.2$ & 57.1 & 41.8 \\
& $\geq 1.4$ & 57.1 & 42.7 \\
& $\geq 1.6$ & 51.4 & 45.6 \\
& $\geq 1.8$ & 22.9 & 71.8 \\
$\geq 2.0$ & 5.7 & 86.7 \\
\hline
\end{tabular}

improved specificity because, depending on the cost and intensity of services targeted to these patients, a false positive rate of $64.1 \%$ achieved using only the OASIS function domain may not be acceptable.

Study findings may guide future research by identifying patients in need of further screening. An agency could use an OASIS function score $\geq 2$ to identify those patients with whom they would like to use the $\mathrm{P}_{\mathrm{ra}}$. Employing the $\mathrm{P}_{\mathrm{ra}}$ in this manner would increase efficiency by starting off with a set of patients likely to score greater than .50 on the $P_{\text {ra. }}$. Subsequent use of the $P_{r a}$ would increase the ability to identify patients at risk and in need of targeted interventions. The range of $\mathrm{P}_{\mathrm{ra}}$ scores could guide stratification of patients into risk groups (e.g., .50-.75, moderate risk, .76-100, high risk). These patients could receive more intense home care or the specialized services of advanced practice nurses to perhaps break the disruptive cycle of readmissions back and forth between home care and acute care.

This study was limited to patients admitted to one home care agency in southeastern Pennsylvania with $\mathrm{CHF}$ as a comorbid condition and may not reflect the general population of CHF or home care patients. The time frame for rehospitalization was limited to 60 days, which may not be the ideal length of time for capturing the most accurate prediction. The $\mathrm{P}_{\mathrm{ra}}$ was designed to predict risk of rehospitalization for a period of 4 years. Perhaps our duration of 60 days did not adequately match the duration most sensitive to prediction by the $\mathrm{P}_{\mathrm{ra}}$, and a longer period is needed to improve accuracy. 
However, Anderson et al. (1999) suggested that 2-3 weeks after home care admission is the most common time for rehospitalization, and our time frame was even longer than that period. Finally, a subsequent study using a larger sample could be helpful in refining the estimates of predictive accuracy, resulting in a more concise confidence interval around the AUC estimates.

Both instruments are helpful in predicting which patients are likely to be rehospitalized. Clinicians might apply the information by performing a more frequent or comprehensive assessment for the early symptoms of heart failure in patients who score more than 2 in the function area of the OASIS and .50 or higher on the $\mathrm{P}_{\mathrm{ra}}$, if used. They might also target specialized interventions, such as more intense teaching, monitoring, or personal contact with these patients. The sensitivity of both the $P_{r a}$ and OASIS function scores was generally good, underscoring the value of these instruments in helping administrators and clinicians target services to those most in need. This study provides a first attempt to compare the OASIS and $\mathrm{P}_{\text {ra }}$ scores in order to guide clinical decision making in home care. Study findings encourage further research to explore the usefulness of these instruments by analyzing individual OASIS items and varied time frames for rehospitalization, using these findings to guide stratification in future studies, as well as testing the instruments with larger samples and with patients with diagnoses other than congestive heart failure.

\section{REFERENCES}

Anderson, M.A., Helms, L.B., Hanson, K.S., \& DeVilder, N.W. (1999). Unplanned hospital readmissions: A home care perspective. Nursing Research, 48, 299-307.

Berkman, B., Walker, S., Bonander, E., \& Holmes, W. (1992). Early unplanned readmissions to social work of elderly patients: Factors predicting who needs follow-up services. Social Work in Health Care, 17, $103-119$.

Boult, C., Dowd, B., McCaffrey, D., Boult, L., Hernandez, R., \& Krulewitch, H. (1993). Screening elders for risk of hospital admission. Journal of the American Geriatrics Society, 41, 811-817.

Boult, L., Boult, C., Pirie, P., \& Pacala, J.T. (1994). Testretest reliability of a questionnaire that identifies elders at risk for hospital admission. Journal of the American Geriatrics Society, 42, 707-711.

Boult, C., Pacala, J.T., \& Boult, L.B. (1995). Targeting elders for geriatric evaluation and management: Reliability, validity, and practicality of a questionnaire. Aging, 7, 159-164.
Bowles, K.H., Naylor, M.D., \& Foust, J.B. (2002). Patient characteristics at hospital discharge and a comparison of home care referral decisions. Journal of the American Geriatrics Association, 50, 336342 .

Brennan, P.F., \& Hays, B.J. (1992). The kappa statistic for establishing interrater reliability in the secondary analysis of qualitative clinical data. Research in Nursing \& Health, 15, 153-158.

Burns, R., \& Nichols, L.O. (1991). Factors predicting readmission of older general medicine patients. Journal of General Internal Medicine, 6, 389-393.

Corrigan, J.M., \& Martin, J.B. (1992). Identification of factors associated with hospital readmission and development of a predictive model. Health Services Research, 27(1), 81-101.

DeLong, E.R., DeLong, D.M., \& Clarke-Pearson, D.L. (1988). Comparing the areas under two or more correlated receiver operating characteristic curves: A nonparametric approach. Biometrics, 44, 837845.

Flaherty, J.H., Perry, H.M., Lynchard, G.S., \& Morley, J.E. (2000). Polypharmacy and hospitalization among older home care patients. Journals of Gerontology Series A: Biological Sciences and Medical Sciences, 55, M554-559.

Fleiss, J.L. (1986). The design and analysis of clinical experiments. New York: Wiley.

Fortinsky R.H. and Madigan, E.A. (1997). Home care resource consumption and patient outcomes: What are the relationships? Home Health Services Quarterly, 16, 55-73.

Fortinsky, R.H., Madigan, E.A., \& Tullai-McGuiness, S. (2000). Resource use and patient outcomes in Medicare home care. Caring, 19(11), 20-21.

Happ, M.B., Naylor, M.D., \& Roe-Prior, P. (1997). Factors contributing to rehospitalization of elderly patients with heart failure. Journal of Cardiovascular Nursing, 11(4), 75-84.

Hosmer, D.W., \& Lemeshow, S. (1989). Applied logistic regression. New York: Wiley.

Hoskins, L.M., Walton-Moss, B., Clark, H.M., Schroeder, M.A., \& Thiel, L. (1999). Predictors of hospital admission among the elderly with congestive heart failure. Home Healthcare Nurse, 17, 373381 .

Kellogg, F.R., Brickner, P.W., Conley, L., \& Conroy, M. (1991). Controlling hospital readmission of elderly persons living at home: A risk factor analysis. Home Health Care Services Quarterly, 12(2), 5-16.

Kerr, M.E. (1994). How reliable are your reliability measures? In R.M. Carroll-Johnson \& M. Paquette (Eds.), Classification of nursing diagnosis: Proceedings of the tenth conference (pp. 291-293). Philadelphia: Lippincott.

Lagoe, R.J., Noetscher, C.M., \& Murphy, M.P. (2001). Hospital readmission: Predicting the risk. Journal of Nursing Care Quality, 15(4), 69-83.

Leiby, S.A., \& Shupe, D.R. (1992). Does home care lessen hospital readmissions for the elderly? Home Healthcare Nurse, 10, 37-42. 
Madigan, E.A., \& Fortinsky, R.H. (2000). Additional psychometric evaluation of the outcomes and assessment information set (OASIS). Home Health Care Services Quarterly, 18(4), 49-62.

Martens, K.H., \& Mellor, S.D. (1997). A study of the relationship between home care services and hospital readmission of patients with congestive heart failure. Home Healthcare Nurse, 15, 123-129.

McNeil, B.J., Keeler, E., \& Adelstein, S.J. (1975). Primer on certain elements of medical decision making. New England Journal of Medicine, 293, 211-215.

Naylor, M.D., Brooten, D., Campbell, R., Jacobsen, B.S., Mezey, M., Pauly, M., et al. (1999). Comprehensive discharge planning and home follow-up of hospitalized elders. Journal of the American Medical Association, 281, 613-620.

Naylor, M., Brooten, D., Jones, R., Lavizzo-Mourey, R., Mezey, M., \& Pauly, M. (1994). Comprehensive discharge planning for hospitalized elderly. A randomized clinical trial. Annals of Internal Medicine, 120, 999-1006.

Neal, L.J. (2000). OASIS inter-rater reliability. Caring, 19(8), 44-47.

Nunnally, J.C., \& Bernstein, I.H. (1994). Psychometric theory (2nd ed.) New York: McGraw-Hill.

Obuchowski, N.A. (1994). Computing sample size for receiver operating characteristic studies. Investigative Radiology, 29, 238-242.

Pacala, J.T., Boult, C., \& Boult, L. (1995). Predictive validity of a questionnaire that identifies older persons at risk for hospital admission. Journal of the American Geriatrics Society, 4, 374-377.

Pacala, J.T., Boult, C., Reed, R.L., \& Aliberti, E. (1997). Predictive validity of the $\mathrm{P}_{\mathrm{ra}}$ instrument among older recipients of managed care. Journal of the American Geriatrics Society, 45, 614-617.

Proctor, E.K., Morrow-Howell, N., Li, H., \& Dore, P. (2000). Adequacy of home care and hospital read- mission for elderly congestive heart failure patients. Health and Social Work, 25(2), 87-96.

Redeker, N.S., \& Brassard, A.B. (1996). Health patterns of cardiac surgery clients using home health care nursing services. Public Health Nursing, 13, 394 403.

Reed, R., Buckner, D., \& Pearlman, R. (1991). Risk factors for early unplanned readmission in the elderly. Journal of General Internal Medicine, 6, 223-228.

Shaughnessy, P.W., Crisler, K.S., \& Schlenker, R.E. (1998). Outcome based quality improvement in home health care: The OASIS indicators. Home Health Care Management and Practice, 10(2), 11-19.

Shaughnessy, P.W., Schlenker, R.E., \& Hittle, D.F. (1995). Case mix of home health patients under capitated and fee-for-service payment. Health Services Research, 30, 79-113.

Shipton, S. (1996). Risk factors associated with multiple hospital readmissions. Home Care Provider, $1,83-85$.

Shipton, S. (1997). Congestive heart failure readmission. Home Care Provider, 2, 171-175.

Soeken, K.L., Prescott, P.A., Herron, D.G., \& Creasia, J. (1991). Predictors of hospital readmission. A metaanalysis. Evaluation and the Health Professions, 14, 262-281.

Soz, H.C., Blatt, M.A., Higgins, M.C., \& Marion, K.I. (1988). Medical decision making. Boston: Butterworth-Heinemann.

Vinson, J., Rich, M., Sperry, J., Shah, A., \& McNamara, T. (1990). Early readmission of elderly patients with congestive heart failure. Journal of the American Geriatrics Society, 38, 1291-1295.

Winograd, C.H., Gerety, M.B., Chung, M., Goldstein, M.K., Dominguez, F., \& Vallone, R. (1991). Screening for frailty: Criteria and predictors of outcomes. Journal of the American Geriatrics Society, 39, $778-784$. 